\title{
Practical Throw-Back Interpolation
}

\author{
By F. D. Burgoyne
}

\begin{abstract}
Precise conditions are determined for the validity of some frequently-used throwback interpolation formulae.
\end{abstract}

While there exist some very powerful throw-back interpolation techniques, such as the Everett-Bessel-Chebyshev formula [1], yet it is probably true that the majority of practical throw-back interpolation makes use of either the simple modified Bessel formula

$$
f_{p} \simeq f_{0}+p \delta f_{1 / 2}+\frac{1}{2}\left(\begin{array}{l}
p \\
2
\end{array}\right)\left(\delta^{2}+c \delta^{4}\right)\left(f_{0}+f_{1}\right)+\frac{1}{3}\left(p-\frac{1}{2}\right)\left(\begin{array}{l}
p \\
2
\end{array}\right) \delta^{3} f_{1 / 2},
$$

or the corresponding Everett formula

$$
f_{p} \simeq f_{0}+p \delta f_{1 / 2}-\left(\begin{array}{l}
p \\
3
\end{array}\right)\left(\delta^{2}+c \delta^{4}\right) f_{0}-\left(\begin{array}{l}
q \\
3
\end{array}\right)\left(\delta^{2}+c \delta^{4}\right) f_{1}
$$

where $c=-0.184$ and $q=1-p$, see [2].

We do not here propose to add anything to the general theory of the subject, which has been developed by Comrie [3], extended by Miller [4], and rounded off by Kopal [5] and Fox [1]. Since, however, (1) and (2) are so frequently used, in this article we determine precise conditions for them to be valid, assuming that differences above a certain order are negligible. Other formulae using differences of the same order, including the rarely-used modified Stirling formula [6], may be examined in a similar way. Formulae using higher differences may, in principle, be dealt with by an extension of the procedure described here, although a prohibitive amount of computation and tabulation would be involved.

When (1) or (2) is used, it is frequently assumed that fifth and higher differences are negligible. We shall examine this situation, and we will also deal with the more interesting case where fifth differences have to be considered, but sixth and higher differences are negligible. Such formulae are usually considered valid if the maximum absolute error is $<\frac{1}{2}$ unit, and this is the criterion we shall use here.

If fifth and higher differences are negligible, fourth differences are constant, and we will write $\delta^{4} f_{0}=\delta^{4} f_{1}=\delta^{4} f$. In this case, the error of (1) is plainly

$$
\left\{-\left(\begin{array}{c}
p+1 \\
4
\end{array}\right)+c\left(\begin{array}{l}
p \\
2
\end{array}\right)\right\} \delta^{4} f
$$

while that of (2) is

$$
\left\{\left(\begin{array}{c}
p+1 \\
5
\end{array}\right)+\left(\begin{array}{c}
q+1 \\
5
\end{array}\right)-c\left(\begin{array}{l}
p \\
3
\end{array}\right)-c\left(\begin{array}{l}
q \\
3
\end{array}\right)\right\} \delta^{4} f=\left\{-\left(\begin{array}{c}
p+1 \\
4
\end{array}\right)+c\left(\begin{array}{l}
p \\
2
\end{array}\right)\right\} \delta^{4} f
$$

Received January 30, 1970, revised April 13, 1970.

AMS 1969 subject classifications. Primary 6520; Secondary 6505, 6510.

Key words and phrases. Throw-back interpolation, precise conditions for validity, Bessel formula, Everett formula, fifth differences negligible, sixth differences negligible.

Copyright @ 1971, American Mathematical Society 
also. Hence, in each case, we require that

$$
\max _{0 \leqq p \leqq 1}\left|-\left(\begin{array}{c}
p+1 \\
4
\end{array}\right)+c\left(\begin{array}{l}
p \\
2
\end{array}\right)\right|\left|\delta^{4} f\right|<\frac{1}{2} \text { unit }
$$

for the formula to be valid. Since

$$
\max _{0 \leqq p \leqq 1}\left|-\left(\begin{array}{c}
p+1 \\
4
\end{array}\right)+c\left(\begin{array}{l}
p \\
2
\end{array}\right)\right| \simeq 0.0004507,
$$

we therefore require that $\left|\delta^{4} f\right| \leqq 1109$ units.

If fifth differences are not negligible, no such simple result exists. Moreover, in this case, the error of (1) is different from that of (2). It is shown in [1] that the maximum absolute error of (1) is approximately $0.00045\left|\mu \delta^{4} f_{1 / 2}\right|+0.00087\left|\delta^{5} f_{1 / 2}\right|$, while that of (2) is only approximately $0.00045\left|\mu \delta^{4} f_{1 / 2}\right|+0.00061\left|\delta^{5} f_{1 / 2}\right|$. It is also pointed out that for practical reasons (2) is the more convenient formula. In these circumstances, we discard (1) in favour of (2), although the same method could be used to derive results for either.

If sixth and higher differences are negligible, the maximum absolute error of (2) is

$$
g(x, y)=\max _{0 \leqq p \leqq 1}\left|\left\{\left(\begin{array}{c}
p+1 \\
5
\end{array}\right)-c\left(\begin{array}{l}
p \\
3
\end{array}\right)\right\} x+\left\{\left(\begin{array}{c}
q+1 \\
5
\end{array}\right)-c\left(\begin{array}{l}
q \\
3
\end{array}\right)\right\} y\right|,
$$

where $x=\delta^{4} f_{0}$ and $y=\delta^{4} f_{1}$. We note, in passing, that $g(-x,-y)=g(x, y)$ and $g(y, x)=g(x, y)$.

We may find various upper bounds for $g(x, y)$ and from them determine permissible values of $x$ and $y$ for (2) to be valid. While this is the approach normally adopted, yet it tends to give rather conservative results. Firstly, we have

$$
\begin{aligned}
g(x, y) & \leqq \max _{0 \leqq p \leqq 1}\left\{\left|\left(\begin{array}{c}
p+1 \\
5
\end{array}\right)-c\left(\begin{array}{l}
p \\
3
\end{array}\right)\right|+\left|\left(\begin{array}{c}
q+1 \\
5
\end{array}\right)-c\left(\begin{array}{l}
q \\
3
\end{array}\right)\right|\right\} \max (|x|,|y|) \\
& \simeq 0.0012160 \max (|x|,|y|) .
\end{aligned}
$$

This shows that (2) is certainly valid if $|x|,|y| \leqq 411$ units. This is the condition most frequently given; we shall see shortly how conservative it is. Secondly, we have

$$
g(x, y) \leqq \max _{0 \leqq p \leqq 1}\left|\left(\begin{array}{c}
p+1 \\
5
\end{array}\right)-c\left(\begin{array}{l}
p \\
3
\end{array}\right)\right|(|x|+|y|) \simeq 0.0007948(|x|+|y|) .
$$

This shows that (2) is valid if $|x|+|y| \leqq 629$ units. Again, this is conservative.

We now describe a tabulation which has been carried out on the University of London CDC 6600 computer, which gives, for each possible value of $x$, the range of values of $y$ for which $g(x, y)<\frac{1}{2}$ unit. A small part of the tabulation is reproduced below.

\begin{tabular}{rrrrrrrrr}
\multicolumn{1}{c}{$u$} & $v$ & $w$ & $u$ & $v$ & $w$ & $u$ & $v$ & \multicolumn{1}{c}{$w$} \\
0 & -629 & 629 & 400 & -417 & 814 & 800 & 369 & 984 \\
100 & -579 & 677 & 500 & -252 & 857 & 900 & 599 & 1025 \\
200 & -528 & 724 & 600 & -59 & 900 & 1000 & 839 & 1066 \\
300 & -474 & 769 & 700 & 149 & 943 & 1100 & 1085 & 1106
\end{tabular}


If $x=u$, we require that $v \leqq y \leqq w$. The tabulation also shows that if $x=-u$, we require that $-w \leqq y \leqq-v$, if $y=u$, we require that $v \leqq x \leqq w$, and if $y=-u$, we require that $-w \leqq x \leqq-v$. These last three results also follow from the fact that $g(x, y)=g(-x,-y)=g(y, x)=g(-y,-x)$.

In the $(x, y)$-plane, the relation $g(x, y)<\frac{1}{2}$ defines a diamond-shaped region, convex outwards, which is symmetrical about the origin and the line $x=y$.

Department of Mathematics

University of London King's College

Strand

London W. C. 2, England

1. L. Fox, Mathematical Tables, Vol. 1, H.M.S.O., London, 1956.

2. Interpolation and Allied Tables, H.M.S.O., London, 1956.

3. L. J. ComRIE, British Association Mathematical Tables. Vol. 1, Cambridge Univ. Press, Cambridge, 1931.

4. J. C. P. Miller, "Two numerical applications of Chebyshev polynominals," Proc. Roy. Soc. Edinburgh Sect. A, v. 62, 1946, pp. 204-210. MR 8, 172.

5. Z. KoPAL, Numerical Analysis, Wiley, New York, 1955. MR 17, 1007.

6. G. J. LidSTONE, "Notes on interpolation," J. Inst. Actuar., v. 71, 1941, pp. 68-95. MR 2, 367. 